\title{
Efficacy of Live $Z$-Score Neurofeedback Training for Chronic Insomnia: A Single-Case Study
}

\author{
Rubén Pérez-Elvira', José A. Carrobles², Diego J. López Bote ${ }^{1}$, and Javier Oltra-Cucarella ${ }^{3^{*}}$ \\ ${ }^{1}$ NEPSA Rehabilitación Neurológica, Salamanca, Spain \\ 2Departamento de Personalidad, Evaluación y Tratamientos Psicológicos, Universidad Autónoma de Madrid, Madrid, \\ Spain \\ ${ }^{3}$ Departamento de Psychología de la Salud, Universidad de Alicante, Alicante, Spain
}

\begin{tabular}{|c|c|}
\hline \multicolumn{2}{|c|}{ Abstract } \\
\hline \multicolumn{2}{|c|}{$\begin{array}{l}\text { Objective/Background: Insomnia is the most common sleep disorder in the general population. } \\
\text { Pharmacological treatments have shown efficacy in the short term, yet the symptoms return once the treatment } \\
\text { has been withdrawn. In the search for treatment options with long-lasting effects, neurofeedback (NF) has arisen } \\
\text { as a therapeutic option. Neurofeedback is the application of operant conditioning to brain activity. The aim of } \\
\text { this work is to show the effectiveness of Live Z-Score NF training (LZT), a paradigm within the field of NF, in a } \\
\text { case of insomnia. Participants: A 32-year-old male with chronic insomnia since his adolescence. Methods: } \\
\text { Thirty 35-min sessions of qEEG-guided LZT using patient's highly preferred feedback. The main outcomes of } \\
\text { this study were the patient's qEEG metrics and a visual analog scale of sleep quality throughout the intervention. } \\
\text { Results: qEEG-guided LZT showed an improvement of } 90.63 \% \text { of the patient's qEEG metrics and an } 82.55 \% \\
\text { relief of the clinical symptoms after } 30 \mathrm{NF} \text { sessions. Conclusions: Although more research is needed to } \\
\text { establish that NF based on Live Z-Score is effective for insomnia, our results suggest that NF might be a } \\
\text { therapeutic alternative for the treatment of insomnia. }\end{array}$} \\
\hline \multirow{2}{*}{\multicolumn{2}{|c|}{$\begin{array}{l}\text { Keywords: insomnia; neurofeedback; z-score; qEEG; z-score neurofeedback } \\
\text { Citation: Pérez-Elvira, R., Carrobles, J. A., López Bote, D. J., \& Oltra-Cucarella, J. (2019). Efficacy of Live Z-Score neurofeedback training } \\
\text { for chronic insomnia: A single-case study. NeuroRegulation, 6(2), 93-101. https://doi.org/10.15540/nr.6.2.93 }\end{array}$}} \\
\hline & \\
\hline $\begin{array}{l}\text { *Address correspondence to: Javier Oltra-Cucarella, } \\
\text { Departamento de Psicología de la Salud, Universidad de Alicante, } \\
\text { Campus de San Vicente del Raspeig - 03690 San Vicente del } \\
\text { Raspeig, Alicante, Spain. Email: javier.oltra@ua.es }\end{array}$ & $\begin{array}{l}\text { Edited by: } \\
\text { Rex L. Cannon, PhD, SPESA Research Institute, Bloomfield Hills, } \\
\text { Michigan, USA; Knoxville Neurofeedback Group, Knoxville, } \\
\text { Tennessee, USA }\end{array}$ \\
\hline $\begin{array}{l}\text { Copyright: @ 2019. Pérez-Elvira et al. This is an Open Access article } \\
\text { distributed under the terms of the Creative Commons Attribution } \\
\text { License (CC-BY). }\end{array}$ & $\begin{array}{l}\text { Reviewed by: } \\
\text { Randall Lyle, PhD, Mount Mercy University, Cedar Rapids, lowa, } \\
\text { USA } \\
\text { Nancy L. Wigton, PhD, Grand Canyon University, Phoenix, Arizona, } \\
\text { USA }\end{array}$ \\
\hline
\end{tabular}

\section{Introduction}

About $20 \%$ to $30 \%$ of adults have some type of sleep disorders (Daley, Morin, LeBlanc, Grégoire, \& Savard, 2009; Hammer, Colbert, Brown, \& Ilioi, 2011). Insomnia is the most prevalent sleep disorder (Martínez Hernández, Lozano Olivares, \& Álamo González, 2016), with around $21 \%$ of the population having at least one symptom of insomnia (Ohayon \& Sagales, 2010) and between $6 \%$ and $10 \%$ of the general population presenting the full clinical syndrome of insomnia (Martínez Hernández et al., 2016). Pharmacological treatment for insomnia usually involves the use of benzodiazepines and antidepressants (NIH, 2005). Pharmacological treatments for insomnia have shown efficacy during the first 6 months after the treatment is implemented, with a worsening of sleep quality after treatment withdrawal (Hammer et al., 2011). This lack of longterm effectiveness of pharmacological therapies has highlighted the need to search for pharmacological and nonpharmacological therapy combinations able to maintain clinical improvements. Indeed, the combination of therapies seems to be more beneficial than monotherapy (Hammer et al., 2011).

Regarding nonpharmacological therapies for insomnia, previous studies have analyzed neurofeedback (NF) interventions either as monotherapy or in combination with other therapies. 
The findings reported in these studies suggest that NF could be beneficial to improve sleep quality (Hammer et al., 2011), to maintain long-term improvements, to reduce falling asleep latency (Hoedlmoser et al., 2008) and to reduce awakenings during sleep (Cortoos, De Valck, Arns, Breteler, \& Cluydts, 2010).

NF is a specialized field of biofeedback focused on the electroencephalographic (EEG) activity control (Carrobles, 2016), based on operant conditioning applications to EEG activity. Operant conditioning is a learning procedure that relies on the use of reinforcement to increase the likelihood of a target response (Skinner, 1938). Rewards are stimuli that are a thing of value to the organism and vary in degree across population. In NF training, the targeted response consists of prespecified patterns of brain waves, and the patient is given the reinforcement every time his or her brain waves match the prespecified pattern (Chapin \& RussellChapin, 2014).

The EEG activity is recorded by an amplifier and processed with specialized software that allows the breakdown of the EEG into frequency bands, and also to quantify the mean voltage or amplitude of each band in a specific moment (Carrobles, 2016; Demos, 2005). Through the application of NF, it is possible to reinforce, inhibit, or ignore the different bands. For bands that are being reinforced, an amplitude threshold is established that must be exceeded to obtain feedback; for bands that are being inhibited, a threshold is established under which their amplitudes must remain in order to obtain reinforcement. When more than one frequency band is being reinforced and/or inhibited, all set thresholds must be within the range set to receive feedback (Demos, 2005). This feedback, which can be visual (e.g., films, animations), auditory (e.g., music) or mixed, is contingent on the fulfilment of the thresholds for each band in the EEG. The clinician usually selects the amplitude thresholds for each frequency band in order to ensure that the patient receives feedback at least $50 \%$ of the time, although learning can take place even with feedback percentages between $20 \%$ and $70 \%$ (Soutar \& Longo, 2011).

Previous studies have shown that the application of NF has beneficial effects in patients with sleep problems (Arns, Feddema, \& Kenemans, 2014; Arns \& Kenemans, 2014; Halson, 2017; Hammer et al., 2011; Schabus et al., 2014), while others have not shown superior effects compared to placebo (Schabus et al., 2017). However, an important limitation related to the NF protocol is found across these studies. The works by Arns et al. (2014), Arns and Kenemans (2014), Halson et al. (2017), and Schabus et al. $(2017,2014)$ assessed the effects of NF on the sensorimotor rhythm (SMR), a brain wave with a frequency in the range of 13 to $15 \mathrm{~Hz}$ that is recorded over the sensorimotor cortex (Arroyo et al., 1993). The SMR protocol was developed in the first place for the treatment of epilepsy and was later applied as a one-size-fits-all procedure for other conditions including attention-deficit/hyperactivity disorder (ADHD) or insomnia. In the SMR protocol, brain waves are recorded in $\mathrm{C} 3$ or $\mathrm{Cz}$ sites, and reinforcement are provided when SMR amplitude is increased irrespective of other brain waves in other sites. This means that the SMR protocol is not administered based on the individual's qEEG profile (Cortoos et al., 2010; Hoedlmoser et al., 2008; Schabus et al., 2017, 2014), as waves at different brain sites are not trained.

Hammer et al. (2011) compared a group receiving $Z$-score training guided by qEEG with a group receiving $Z$-score SMR training. As participants receiving Z-score SMR training showed large movements toward Z-score normalization, these authors concluded that the $Z$-score training was probably more related to improvements in sleep quality than was the SMR element and the rewarding of SMR itself.

The technological advances in recent years have allowed an increase in the possibilities of the NF (Hammer et al., 2011) through different paradigms: $Z$-score-based neurofeedback, infralow frequency neurofeedback, infraslow fluctuation neurofeedback, or low-resolution electromagnetic tomography analysis (LORETA)-based neurofeedback. Some authors (Krigbaum \& Wigton, 2015; Lubar, 2015; Wigton \& Krigbaum, 2015) indicate that NF based on Live Z-Score (LZT) produces faster learning than conventional NF and has shown efficacy in different pathologies such as ADHD, epilepsy, migraine, depression, anxiety, and learning disorders (Guan, 2016; Walker, 2016). Hammer et al. (2011) used a 4-channel LZT neurofeedback protocol based on the qEEG results and showed that it might be effective in improving both overall sleep quality and quantity in individuals with insomnia. However, studies on the application of LZT in insomnia are scarce, both in group designs and in single-case designs. The main objective of LZT is to train patient EEG Z-scores, deviated from the norm, to behave normally; it does so with a low probability that waves with a normal amplitude move out of the normal range (Pérez-Elvira et al., 2018). To do this, all the patient's EEG $Z$-scores are computed and collected at all times, the 
percentage of $Z$-scores within a specific range $( \pm 1$ standard deviation $S D$ ) is calculated, and the patient receives feedback every time the percentage of $Z$-scores within the normal range is equal to or higher than the requested percentage.

The use of LZT is aimed at normalizing extreme $Z$-scores, while outliers are avoided so as to not overtrain them. Thus, the brain has sufficient freedom to choose a path of self-regulation that is not limited to training towards the norm (Collura, Guan, Tarrant, Bailey, \& Starr, 2010). In other words, the brain accommodates itself to normalize with a certain degree of flexibility, since the main objective is to place a percentage of waves within the normal range.

Amplitude NF allows for the training of a small number of targets at the same time and does not permit the safe increase of slow waves, such as delta (Soutar \& Longo, 2011). With LZT up to 248 simultaneous $Z$-scores (if four EEG channels are used) can be trained at the same time, and delta waves can be raised with a good safety margin since the limit is a normed Z-score, which is not the case with NF amplitude (Collura et al., 2010; Gracefire, 2016). With LZT the clinician can read the patient's qEEG deviations in real time and adjust the reinforcement thresholds to optimize the intervention.

There is a scarcity of LZT literature published in peerreviewed scientific journals (with institutional review board coverage) reporting targeted qEEG change (Krigbaum \& Wigton, 2015; Wigton \& Krigbaum, 2015). To our knowledge, only one previous study used LZT for insomnia (Hammer et al., 2011). Therefore, the aim of this study is to analyze the efficacy of LZT guided by qEEG in the treatment of chronic insomnia.

\section{Methods}

\section{Patient}

The patient was a 32-year-old male, adopted when he was eight. He suffered from chronic insomnia since his adolescence. He had been receiving psychological treatment from an early age for night terrors and sensory deprivation suffered before his adoption. He started his schooling at eight years of age, because up to that moment he had environmental and psychosocial problems.

When this study began, he was working as a carpenter and was still receiving psychological treatment aimed at improving social skills for problems related to self-esteem. His educational level was primary school.

\section{Main Complaints}

The patient consulted our clinic, looking for a nonpharmacological treatment, because of complaints related to quantity and quality of sleep.

He had problems falling and staying asleep during the night and had frequent and vivid nightmares. The patient was unable to fall asleep, once lying down, until after more than an hour, and woke up frequently during the night without managing to maintain sleep more than 4 hours. Before starting treatment with NF, he received pharmacological treatment for insomnia (quetiapine $100 \mathrm{mg}$ ). Yet he often woke up during the night and felt anxious. This fact conditioned the rest of his day, both at work and in his social life. In addition, the patient expressed a desire to withdraw the medication.

The main objectives of the patient were to (1) fall asleep, to reduce the time between lying down and starting to sleep to less than 20 minutes; (2) stay asleep during most of the night, the total sleep time to be at least 6 hours; (3) feel refreshed after sleeping; and (4) withdraw sleep medication without worsening the quantity and quality of sleep.

The patient provided written informed consent for the intervention and the publication of this study, which was undertaken at NEPSA Rehabilitación Neurológica, a neurologic rehabilitation clinic authorized by the Regional Department of Health (Castilla y León, Spain). The Regional Department of Health provided approval for this kind of intervention.

\section{Instruments and Procedure}

qEEG Recording and Analysis. A qEEG was obtained before starting the NF intervention, and after every $10 \mathrm{NF}$ sessions. To obtain the EEG, the patient was fitted with a 19-channel (Electro-cap International, Eaton, $\mathrm{OH}$ ) according to the International 10-20 System with linked-ear montage (Fp1, Fp2, F7, F3, Fz, F4, F8, T3, C3, Cz, C4, T4, T5, $\mathrm{P} 3, \mathrm{Pz}, \mathrm{P} 4, \mathrm{~T} 6, \mathrm{O} 1$, and O2). For $3 \mathrm{~min}$, the EEG signals were obtained and collected simultaneously over these 19 channels with a Discovery 20 amplifier (BrainMaster Technologies, Inc., Bedford, $\mathrm{OH}$ ). The EEG recordings were recorded in eyes-closed condition, using BrainAvatar 4.6.4 software (BrainMaster Technologies, Inc., Bedford, OH).

The EEG signals were then imported into the NeuroGuide v2.9.1 software (Applied Neuroscience, Inc., Largo, FL) for computation and analysis, where artifacts (i.e., activity collected from the EEG that is not produced by the brain) were visually inspected and removed, retaining $1 \mathrm{~min}$ and $33 \mathrm{~s}$ with a test- 
retest value of 0.93 . The EEG was processed with linked-ear montage and compared with the NeuroGuide normative database, and Z-score values were obtained in order to identify the patient's brain waves that were out of range.

Visual Analog Scale. Prior to starting the NF intervention, the patient rated his sleep quality using a subjective visual scale. The visual scale included a $10 \mathrm{~cm}$ line with numbers from 0 to 100 , with 0 being no sleep problems and 100 being the worst sleep quality. This scale was administered before the NF intervention and after every 10 sessions during the intervention. The upscale was also administered after 1-, 3-, and 6-month follow-ups.

\section{Intervention}

Live Z-Score Training (LZT) Neurofeedback. We used qEEG-guided LZT. Since C3, C4, P3, and P4 were the most deviated waves from normal range at pretreatment, they were selected for NF intervention. We used a 4-channel linked ears montage and the BrainAvatar 4.6.4 Z-Score PZOKUL protocol (BrainMaster Technologies, Inc., Bedford, OH).

This protocol has a training threshold that autoadjusts based on the percentage of $Z$-scores within the upper and lower selected limits. In this study, 1 $S D$ was used as the upper and lower thresholds (Thatcher \& Lubar, 2014). The patient received one 35-min session twice a week for 15 weeks without interruption, with a total of 30 LZT sessions. The patient was offered to choose both the form of feedback (e.g., auditory or visual) and the type of feedback (e.g., sounds, music, videogames, movies, etc.) within each session. According to the patient's preferences, different movies selected by the patient were used to produce the feedback. This procedure is a modification of the procedure used in previous studies where the same form and type of feedback was selected by the researcher and used for all participants. For instance, Schabus et al. (2017, 2014) and Hoedlmoser et al. (2008) used the drawing of a sun along with a sound, whereas other studies did not specify what feedback was used (Cortoos et al., 2010; Hammer et al., 2011). We allowed the patient to choose both the form and the type of feedback in each session because, as previous studies have shown (Fisher et al., 1992; Mangum, Fredrick, Pabico, \& Roane, 2012; Piazza, Fisher, Hagopian, Bowman, \& Toole, 1996), rewards that are more relevant for the subject have a superior learning effect.

A dimmer was placed in front of the video screen that offered sharpness when the patient met the criteria set by the LZT protocol or became opaque, preventing the video from being viewed, when the criteria were not met.

\section{Statistical Analysis}

To analyze the change in EEG values, $Z$-scores were obtained for each location and frequency band, with a total of eight bands (Table 1 ). These $Z$-scores were dichotomized as within (i.e., $\pm 1 S D$ ) or outside the norm. The number of Z-scores within the limits of normality every 10 sessions was calculated. The McNemar test was used for related data to analyze whether significant changes occurred after each block of 10 sessions.

To analyze the clinical change, we used the percentage change of the values of the visual sleep quality scale.

\section{Results}

The pretreatment qEEG showed that all brain waves were under the lower limit for all four locations in all frequency bands (Table 1), mainly in the delta and beta bands, and in central, parietal, and occipital regions (Figure 1). The patient scored 86 on the visual analog sleep quality scale (Figure 2). The percentage of Z-scores within the normal limits was $0 \%$ (Tables 2 and 3 ). 


\section{Z-Scores}
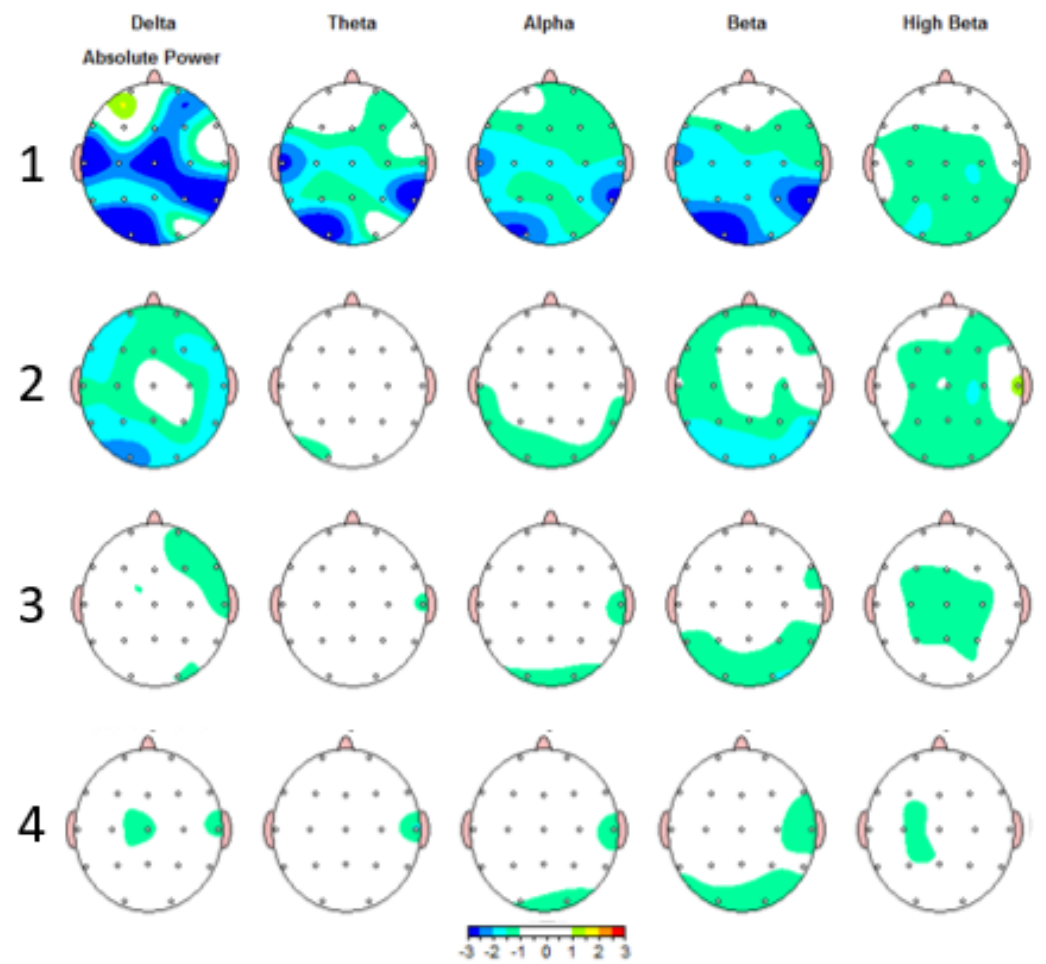

Figure 1. Surface maps of Z-scores distribution (full EEG). 1 = Baseline qEEG, 2 = after $10 \mathrm{LZT}$ sessions qEEG, 3 = after $20 \mathrm{LZT}$ sessions qEEG, 4 = after 30 LZT sessions qEEG.

\begin{tabular}{|c|c|c|c|c|}
\hline \multicolumn{5}{|c|}{$\begin{array}{l}\text { Table } 1 \\
Z \text {-scores per channel and frequency, and } \\
\text { evaluation. }\end{array}$} \\
\hline Channels & $\begin{array}{l}\text { Delta } \\
\text { Initial }\end{array}$ & $\begin{array}{l}\text { Delta } \\
\text { 10NF }\end{array}$ & $\begin{array}{l}\text { Delta } \\
\text { 20NF }\end{array}$ & $\begin{array}{l}\text { Delta } \\
\text { 30NF }\end{array}$ \\
\hline C3 & -2.38 & -1.37 & -0.64 & -0.69 \\
\hline P3 & -1.83 & -1.60 & -0.56 & -0.83 \\
\hline C4 & -1.82 & -0.92 & -0.67 & -0.57 \\
\hline P4 & -2.63 & -0.96 & -0.83 & -0.47 \\
\hline Channels & $\begin{array}{l}\text { Theta } \\
\text { Initial }\end{array}$ & $\begin{array}{l}\text { Theta } \\
\text { 10NFF }\end{array}$ & $\begin{array}{l}\text { Theta } \\
\text { 20NF }\end{array}$ & $\begin{array}{l}\text { Theta } \\
\text { 30NF }\end{array}$ \\
\hline C3 & -1.66 & -0.22 & 0.35 & 0.33 \\
\hline P3 & -1.46 & -0.52 & 0.13 & 0.09 \\
\hline C4 & -1.30 & 0.21 & 0.10 & -0.09 \\
\hline P4 & -1.77 & 0.12 & 0.57 & 0.71 \\
\hline
\end{tabular}

\begin{tabular}{lrrrr}
\hline Channels & $\begin{array}{r}\text { Alpha } \\
\text { Initial }\end{array}$ & $\begin{array}{r}\text { Alpha } \\
\text { 10NF }\end{array}$ & $\begin{array}{r}\text { Alpha } \\
\text { 20NF }\end{array}$ & $\begin{array}{c}\text { Alpha } \\
\text { 30NF }\end{array}$ \\
C3 & -1.64 & -0.73 & -0.24 & -0.36 \\
P3 & -1.46 & -0.90 & -0.59 & -0.56 \\
C4 & -1.47 & -0.69 & -0.41 & -0.33 \\
P4 & -1.65 & -0.71 & -0.58 & -0.44 \\
& & & & \\
Channels & Beta & Beta & Beta & Beta \\
& Initial & $10 \mathrm{NF}$ & $20 \mathrm{NF}$ & $30 \mathrm{NF}$ \\
C3 & -1.80 & -1.19 & -0.57 & -0.47 \\
P3 & -1.91 & -1.43 & -0.98 & -0.72 \\
C4 & -1.65 & -1.08 & -0.78 & -0.91 \\
P4 & -2.19 & -1.22 & -1.08 & -0.94 \\
\hline
\end{tabular}

Note. Initial $=1$ st qEEG, $10 \mathrm{NF}=$ after $10 \mathrm{NF}$ sessions, $20 \mathrm{NF}=$ after $20 \mathrm{NF}$ sessions, $30 \mathrm{NF}=$ after $30 \mathrm{NF}$ sessions. Z-scores out of range are marked in blue ink. 


\begin{tabular}{|c|c|c|c|c|}
\hline Channels & $\begin{array}{r}\text { Hi Beta } \\
\text { Initial }\end{array}$ & $\begin{array}{r}\text { Hi Beta } \\
\text { 10NF }\end{array}$ & $\begin{array}{r}\text { Hi Beta } \\
20 N F\end{array}$ & $\begin{array}{r}\text { Hi Beta } \\
\text { 30NF }\end{array}$ \\
\hline C3 & -1.32 & -1.11 & -1.15 & -1.01 \\
\hline P3 & -1.45 & -1.33 & -1.01 & -0.89 \\
\hline C4 & -1.42 & -1.32 & -1.16 & -0.97 \\
\hline P4 & -1.38 & -1.25 & -1.01 & -0.66 \\
\hline Channels & $\begin{array}{l}\text { Beta1 } \\
\text { Initial }\end{array}$ & $\begin{array}{l}\text { Beta1 } \\
\text { 10NF }\end{array}$ & $\begin{array}{l}\text { Beta1 } \\
20 N F\end{array}$ & $\begin{array}{l}\text { Beta1 } \\
\text { 30NF }\end{array}$ \\
\hline C3 & -1.31 & -0.72 & -0.19 & -0.34 \\
\hline P3 & -1.58 & -1.15 & -0.84 & -0.72 \\
\hline C4 & -1.21 & -0.45 & -0.34 & -0.84 \\
\hline P4 & -1.78 & -0.99 & -0.98 & -0.88 \\
\hline Channels & $\begin{array}{l}\text { Beta2 } \\
\text { Initial }\end{array}$ & $\begin{array}{c}\text { Beta2 } \\
10 N F\end{array}$ & $\begin{array}{l}\text { Beta2 } \\
20 N F\end{array}$ & $\begin{array}{r}\text { Beta2 } \\
\text { 30NF }\end{array}$ \\
\hline C3 & -1.39 & -0.97 & 0.13 & 0.29 \\
\hline P3 & -1.61 & -1.19 & -0.47 & -0.23 \\
\hline C4 & -1.19 & -0.79 & -0.19 & -0.13 \\
\hline P4 & -1.69 & -0.69 & -0.54 & -0.59 \\
\hline Channels & $\begin{array}{l}\text { Beta3 } \\
\text { Initial }\end{array}$ & $\begin{array}{c}\text { Beta3 } \\
\text { 10NF }\end{array}$ & $\begin{array}{l}\text { Beta3 } \\
20 N F\end{array}$ & $\begin{array}{c}\text { Beta3 } \\
\text { 30NF }\end{array}$ \\
\hline C3 & -1.91 & -1.53 & -1.36 & -0.96 \\
\hline P3 & -1.87 & -1.58 & -1.28 & -0.87 \\
\hline C4 & -1.87 & -1.76 & -1.50 & -1.30 \\
\hline P4 & -2.18 & -1.62 & -1.34 & -1.05 \\
\hline
\end{tabular}

Note. Initial $=1$ st qEEG, $10 \mathrm{NF}=$ after $10 \mathrm{NF}$ sessions, $20 \mathrm{NF}=$ after $20 \mathrm{NF}$ sessions, 30NF = after $30 \mathrm{NF}$ sessions. Z-scores out of range are marked in blue ink.

After the first 10 sessions of LZT, 16 of the 32 $Z$-scores $(50 \%)$ were within the normal range (Tables 2 and 3 ), which is a statistically significant change relative to baseline assessment $\left(X^{2}=14.06, p<.001\right)$. Also, the qEEG maps showed an overall improvement (Figure 1), despite maintaining low voltage in all frequency bands. After these $10 \mathrm{LZT}$ sessions, the patient identified a $22.09 \%$ improvement in sleep quality (Figure 2).

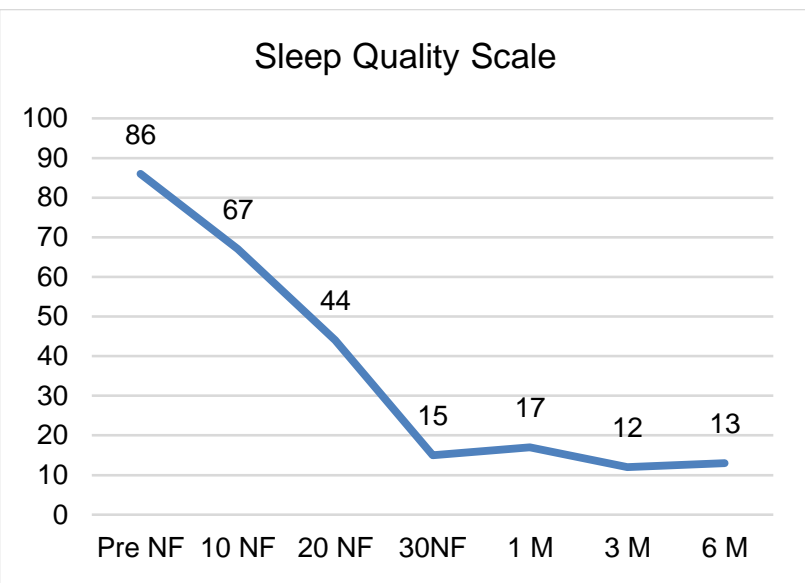

Figure 2. Sleep Quality Visual Analog Scale.

Pretreatment, after 10NF, 20NF, and 30NF sessions, and 1-month (M), $3 \mathrm{M}$, and $6 \mathrm{M}$ follow-up. 100 = worst sleep quality, 0 = best sleep quality.

After 20 LZT sessions, the maps again showed an overall improvement (Figure 1), with higher voltage in all bands and $71.88 \%$ of $Z$-scores within the norm (Tables 2 and 3 ), a significant change compared to the previous measurement $\left(X^{2}=5.14, p=.011\right)$. The patient identified an improvement of $48.83 \%$ compared to the previous assessment on the analogue visual scale (Figure 2) measuring sleep quality.

\begin{tabular}{lrr}
\hline $\begin{array}{l}\text { Table } 2 \\
\text { Percentage of Z-scores within the normal limits for } \\
\text { every measurement distributed by frequency. }\end{array}$ \\
\hline \multicolumn{3}{c}{$\begin{array}{c}\text { Number of } Z \text {-scores } \\
\text { within the limits }\end{array}$} \\
& 0 & $0.00 \%$ \\
Initial & 16 & $50.00 \%$ \\
$10 \mathrm{NF}$ & 23 & $71.88 \%$ \\
$20 \mathrm{NF}$ & 29 & $90.63 \%$ \\
$30 \mathrm{NF}$ & 0
\end{tabular}

Note. Initial $=$ First qEEG, $10 \mathrm{NF}=$ after 10 sessions qEEG, $20 \mathrm{NF}=$ after 20 sessions $\mathrm{qEEG}, 30 \mathrm{NF}=$ after 30 sessions qEEG. 


\begin{tabular}{|c|c|c|c|c|}
\hline $\begin{array}{l}\text { Table } 3 \\
\text { Percentag } \\
\text { every mea }\end{array}$ & $\begin{array}{l}\text { Z-scor } \\
\text { ment c }\end{array}$ & $\begin{array}{l}\text { within th } \\
\text { ibuted }\end{array}$ & $\begin{array}{l}\text { ormal II } \\
\text { channel }\end{array}$ & for \\
\hline $\begin{array}{l}\text { Number of } \\
Z \text {-scores } \\
\text { within the } \\
\text { limits }\end{array}$ & C3 & P3 & C4 & P4 \\
\hline Initial & 0 & 0 & 0 & 0 \\
\hline 10NF & 4 & 2 & 5 & 5 \\
\hline 20NF & 6 & 6 & 6 & 5 \\
\hline $30 N F$ & 7 & 8 & 7 & 7 \\
\hline $\begin{array}{l}\% \text { within } \\
\text { the limits }\end{array}$ & C3 & P3 & C4 & P4 \\
\hline Initial & $0 \%$ & $0 \%$ & $0 \%$ & $0 \%$ \\
\hline $10 \mathrm{NF}$ & $50.0 \%$ & $25.0 \%$ & $62.5 \%$ & $62.5 \%$ \\
\hline $20 N F$ & $75.0 \%$ & $75.0 \%$ & $75.0 \%$ & $62.5 \%$ \\
\hline $30 \mathrm{NF}$ & $87.5 \%$ & $100.0 \%$ & $87.5 \%$ & $87.5 \%$ \\
\hline
\end{tabular}

Note. Initial = First qEEG, $10 N F=$ after 10 sessions qEEG, $20 \mathrm{NF}=$ after 20 sessions $\mathrm{qEEG}, 30 \mathrm{NF}=$ after 30 sessions qEEG.

After 30 LZT sessions, additional improvements were found in the qEEG maps (Figure 1), with higher voltage in all bands and an improvement of $82.5 \%$ in sleep quality compared to the initial measurement (Figure 2). It can be observed that $90.63 \%$ of $Z$-scores were in the normal range after the last LZT session (Tables 2 and 3 ), which is a statistically significant improvement compared to baseline assessment $\left(X^{2}=4.17, p=.02\right)$.

Regarding the channel scores, P3 showed $100 \%$ of the $Z$-scores within the normal range after 30 sessions of NF, whereas C3, C4, and P4 showed $87 \%$ of $Z$-scores within the normal range. As can be seen in Table 1, none of the waves within the normal range deviated from normality (i.e., $\pm 1 S D$ ).

Data on subjective perception of sleep quality after 1-, 3-, and 6-month follow-ups showed no difference compared to the last measurement of the intervention phase, indicating a maintenance of the improvements achieved after 30 sessions of LZT.

\section{Discussion}

This study aimed to analyze the efficacy of LZT neurofeedback intervention for the treatment of chronic insomnia. A qEEG-guided LZT protocol was designed for this purpose. The results showed that LZT was effective in modifying EEG patterns and bringing EEG metrics within the normal range after thirty 35-min sessions of NF. In addition, the patient was able to discontinue the use of sleep medication after 20 sessions.

These results are consistent with previous works. Hammer et al. (2011) found improvements in sleep after 10 LZT sessions in people with insomnia. Krigbaum and Wigton (2015) analyzed 10 individuals with different conditions (attentiondeficit/hyperactivity disorder, anxiety disorder, and Asperger's disorder) using 19-Channel Z-score NF during 6 to $15 \mathrm{NF}$ sessions. They identified the sites of interest as any electrode sites which had $Z$-scores of either $Z \geq 1$ or $Z \leq-1$, and then analyzed those that moved towards values of $Z=0$. Their findings showed that 45 sites of interest out of $50(90 \%)$ moved in the targeted direction, a finding similar to the results reported in the present study using a similar methodology, even though we used the criterion of $Z$-scores falling within the normal range $(Z$-scores between \pm 1 ) rather than moving toward $z=$ 0 .

Our data indicate that after 10 sessions of LZT there was a trend towards normalization of $Z$-scores, and after 30 sessions the total computation of $Z$-scores was very close to normal. Likewise, the patient interrupted the pharmacological treatment and identified an improvement of $83 \%$ in the subjective perception of the quality of sleep. Sleep quality was measured with a visual analog scale during the intervention and in the follow-up, with maintenance of sleep quality at the end of the intervention and withdrawal of the medication. Our results showed a difference of 71 points between baseline and the last NF session. This difference, which is higher than the difference considered clinically relevant by Zisapel and Nir (2003) using a 100 points visual analog scale for measuring quality of sleep, highlights the clinical importance of the LZT and supports the clinical relevance reported in previous works (Hammer et al., 2011; Krigbaum \& Wigton, 2015; Wigton \& Krigbaum, 2015).

One of the strengths of this work is that the effects of NF were assessed both immediately after treatment and after 1-, 3-, and 6-months follow-ups. This is an improvement over other studies which, as far as we know, either did not follow up (Hoedlmoser et al., 2008; Schabus et al., 2017, 2014) or only did it at one time. For instance, Hammer et al. (2011) had a 6- to 9-month follow-up, and Cortoos et al. (2010) had a 
2-week follow-up after the treatment was completed. Interestingly, our results after 6 months of follow-up are similar to those reported by Hammer et al. (2011), who found a good sleep quality in their participants after 6 months of follow-up. Additionally, it must be noted that the improvement in sleep quality in the patient described here continued even after the patient withdrew the medication.

In the present study, qEEG was used to guide treatment with LZT, which allowed specification of the brain waves that would be the sites of interest for the intervention. This could explain the differences with the study by Schabus et al. (2017), who concluded that treatment with NF was not superior to placebo for insomnia. It should be noted that in their study they used the SMR protocol, which was not qEEG-guided and was applied similarly to every participant. Regarding feedback, stimulus used to work as reinforcer for some subjects might not work for others (Mangum et al., 2012). The NF is based on the application of the operant conditioning for the modulation of the EEG activity, so it is important that the feedback is a real reinforcer relevant for the individual patient (Fisher et al., 1992). The feedback used by Schabus et al. (2017) appears to have little probability of being a true reinforcer for the subjects in their study because they were not selected by the subjects and might not work as a reinforcer. It should be added that the results of Schabus et al. (2017) contradict other studies that showed that the use of different NF protocols improved sleep latencies in children and adults with ADHD (Arns et al., 2014; Arns \& Kenemans, 2014). Both the protocol and the feedback used by Schabus et al. (2017) could partially explain their negative results. In contrast, our study used patient's highly preferred feedback, so it is are assumed to have a higher reinforcing value (Fisher et al., 1992; Piazza et al., 1996).

The results of this case suggest the need to investigate the efficacy of LZT not only as a treatment for insomnia but also as a tool to normalize brain activity, including low-voltage cases. Despite our results, this study had numerous limitations, including sample size, which was reduced to a single case without a control group. In the protocol used in the present study, following the fundamental principles of operant conditioning, and in order to ensure that feedback had real reinforcing value, the patient was allowed to choose the feedback to be used. Although this could guarantee the reinforcing value of the material, this procedure makes our results not comparable with previous works that did not allow the patient to select a relevant reinforcer. Also, sleep quality was measured with a visual analog scale, as used in previous research on insomnia (Zisapel \& Nir, 2003), but it probably only reflects very generally the quality of sleep. It is therefore necessary to include objective measures of sleep quality to correlate subjective improvements with objective physiological measures. Similarly, the effect of reinforcer selection on the neurometric results of the intervention with LZT and other types of NF should be analyzed. As for the measures of sleep quality, insomnia is a multicausal pathology and with several dimensions to take into account (e.g., hours of sleep, latency time until conciliation of the same, awakenings during the night). Thus, scales and other instruments are needed that could sufficiently cover the different dimensions of sleep.

In conclusion, LZT seems to be a good approach to NF not only because of its rapid resolution of symptoms and normalization of brain activity but also because of its safety margin for increasing slow waves. In the case of insomnia, LZT may be a better option than pharmacological treatment. As shown by this 30-session intervention, NF may achieve longlasting effects, may normalize the EEG, and may also improve subjective quality of sleep in chronic insomnia, without producing adverse reactions or side effects.

\section{Author Disclosure}

The authors declare that they have no grants, financial interests, or conflicts of interest to disclose.

This work is part of a doctoral thesis by Rubén PérezElvira.

\section{References}

Arns, M., Feddema, I., \& Kenemans, J. L. (2014). Differential effects of theta/beta and SMR neurofeedback in ADHD on sleep onset latency. Frontiers in Human Neuroscience, 8. https://doi.org/10.3389/fnhum.2014.01019

Arns, M., \& Kenemans, J. L. (2014). Neurofeedback in ADHD and insomnia: Vigilance stabilization through sleep spindles and circadian networks. Neuroscience \& Biobehavioral Reviews, 44, 183-194. https://doi.org/10.1016/j.neubiorev.2012.10.006

Arroyo, S., Lesser, R. P., Gordon, B., Uematsu, S., Jackson, D., \& Webber, R. (1993). Functional significance of the mu rhythm of human cortex: an electrophysiologic study with subdural electrodes. Electroencephalography and Clinical Neurophysiology, 87(3), 76-87. https://doi.org/10.1016/00134694(93)90114-B

BrainAvatar (Version 4.6.4) [Computer software]. (n.d.). Bedford, $\mathrm{OH}$ : BrainMaster Technologies, Inc.

BrainMaster Discovery 20 [Apparatus]. Bedford, $\mathrm{OH}$ : BrainMaster Technologies, Inc.

Carrobles, J. A. (2016). Bio/neurofeedback. Clínica y Salud, 27(3), 125-131. https://doi.org/10.1016/j.clysa.2016.09.003

Chapin, T., \& Russell-Chapin, L. A. (2014). Neurotherapy and neurofeedback: Brain-based treatment for psychological and behavioral problems. New York NY: Routledge/Taylor \& Francis Group. 
Collura, T., Guan, J., Tarrant, J., Bailey, J., \& Starr, F. (2010). EEG biofeedback case studies using live $Z$-score training and a normative database. Journal of Neurotherapy, 14(1), 22-46. https://doi.org/10.1080/10874200903543963

Cortoos, A., De Valck, E., Arns, M., Breteler, M. H. M., \& Cluydts, R. (2010). An exploratory study on the effects of teleneurofeedback and tele-biofeedback on objective and subjective sleep in patients with primary insomnia. Applied Psychophysiology and Biofeedback, 35(2), 125-134. https://doi.org/10.1007/s10484-009-9116-z

Daley, M., Morin, C. M., LeBlanc, M., Grégoire, J.-P., \& Savard, J. (2009). The economic burden of insomnia: direct and indirect costs for individuals with insomnia syndrome, insomnia symptoms, and good sleepers. Sleep, 32(1), 55-64.

Demos, J. N. (2005). Getting started with neurofeedback (1st ed). New York: W.W. Norton \& Company.

Electro-Cap system [Apparatus]. Eaton, $\mathrm{OH}$ : Electro-Cap International, Inc.

Fisher, W., Piazza, C. C., Bowman, L. G., Hagopian, L. P., Owens, J. C., \& Slevin, I. (1992). A comparison of two approaches for identifying reinforcers for persons with severe and profound disabilities. Journal of Applied Behavior Analysis, 25(2), 491498. https://doi.org/10.1901/jaba.1992.25-491

Gracefire, P. (2016). Introduction to the concepts and clinical applications of multivariate live Z-Score training, PZOK and sLORETA feedback. In T. F. Collura \& J. A. Frederick (Eds.), Handbook of clinical QEEG and neuropathy (pp. 326-383). New York, NY: Routledge.

Guan, J. (2016). The efficacy of $Z$-score neurofeedback training. In T. F. Collura \& J. A. Frederick (Eds.), Handbook of clinical QEEG and neuropathy (pp. 312-325). New York, NY: Routledge.

Halson, S. L. (2017). Neurofeedback as a Potential Nonpharmacological Treatment for Insomnia. Biofeedback, 45(1), 19-20. https://doi.org/10.5298/1081-5937-45.1.08

Hammer, B. U., Colbert, A. P., Brown, K. A., \& llioi, E. C. (2011). Neurofeedback for insomnia: A pilot study of Z-Score SMR and individualized protocols. Applied Psychophysiology and Biofeedback, 36(4), 251-264. https://doi.org/10.1007/s10484011-9165-y

Hoedlmoser, K., Pecherstorfer, T., Gruber, G., Anderer, P., Doppelmayr, M., Klimesch, W., \& Schabus, M. (2008). Instrumental conditioning of human sensorimotor rhythm (12$15 \mathrm{~Hz}$ ) and its impact on sleep as well as declarative learning. Sleep, 31(10), 1401-1408. http://doi.org/10.5665/sleep /31.10.1401

Krigbaum, G., \& Wigton, N. L. (2015). A methodology of analysis for monitoring treatment progression with 19-channel Z-score neurofeedback (19ZNF) in a single-subject design. Applied Psychophysiology and Biofeedback, 40(3), 139-149. https://doi.org/10.1007/s10484-015-9274-0

Lubar, J. F. (2015). Optimal procedures in Z-score neurofeedback. In R. W. Thatcher \& D. S. Foster (Eds.), Z score neurofeedback: Clinical applications (pp. 41-58). San Diego, CA: Academic Press. https://doi.org/10.1016/B978-0-12801291-8.00003-0

Mangum, A., Fredrick, L., Pabico, R., \& Roane, H. (2012). The role of context in the evaluation of reinforcer efficacy: Implications for the preference assessment outcomes. Research in Autism
Spectrum Disorders, 6(1), 158-167. https://doi.org/10.1016/j.rasd.2011.04.001

Martínez Hernández, J., Lozano Olivares, J., \& Álamo González, C. (2016). Insomnio. Madrid, Spain: FFOMC IM\&C.

NeuroGuide (Version 2.9.1) [Computer software]. (n.d.). Largo, FL: Applied Neuroscience, Inc.

$\mathrm{NIH}$. (2005). NIH State-of-the-Science Conference Statement on manifestations and management of chronic insomnia in adults. $\mathrm{NIH}$ Consensus and State-of-the-Science Statements, 22(2), $1-30$.

Ohayon, M. M., \& Sagales, T. (2010). Prevalence of insomnia and sleep characteristics in the general population of Spain. Sleep Medicine, 11(10), 1010-1018. https://doi.org/10.1016 /j.sleep.2010.02.018

Pérez-Elvira, R., López Bote, D. J., Guarino, S., Agudo Juan, M., De León, R. J., Feiner, T., \& Perez, B. (2018). Neurometric results of a case series using live $Z$-scores neurofeedback. International Journal of Psychophysiology, 131, S139-S140. https://doi.org/10.1016/j.ijpsycho.2018.07.375

Piazza, C. C., Fisher, W. W., Hagopian, L. P., Bowman, L. G., \& Toole, L. (1996). Using a choice assessment to predict reinforcer effectiveness. Journal of Applied Behavior Analysis, 29(1), 1-9. https://doi.org/10.1901/jaba.1996.29-1

Schabus, M., Griessenberger, H., Gnjezda, M.-T., Heib, D. P. J., Wislowska, M., \& Hoedlmoser, K. (2017). Better than sham? A double-blind placebo-controlled neurofeedback study in primary insomnia. Brain, 140(4), 1041-1052. https://doi.org /10.1093/brain/awx011

Schabus, M., Heib, D. P. J., Lechinger, J., Griessenberger, H., Klimesch, W., Pawlizki, A., ... Hoedlmoser, K. (2014). Enhancing sleep quality and memory in insomnia using instrumental sensorimotor rhythm conditioning. Biological Psychology, 95, 126-134. https://doi.org/10.1016 /j.biopsycho.2013.02.020

Skinner, B. F. (1938). The behavior of organisms: An experimental analysis. New York, NY: Appleton-Century-Crofts, Inc.

Soutar, R. G., \& Longo, R. E. (2011). Doing neurofeedback: An introduction (pp. 121-140). ISNR Research Foundation.

Thatcher, R. W., \& Lubar, J. F. (Eds.). (2014). Z score neurofeedback: Clinical applications. San Diego, CA: Academic Press.

Walker, J. E. (2016). qEEG-guided neurofeedback to normalize brain function in various disorders. In T. F. Collura \& J. A Frederick (Eds.), Handbook of clinical QEEG and neuropathy (pp. 149-157). New York, NY: Routledge.

Wigton, N. L., \& Krigbaum, G. (2015). Attention, executive function, behavior, and electrocortical function, significantly improved with 19-channel $z$-score neurofeedback in a clinical setting: A pilot study. Journal of Attention Disorders, 23(4), 398-408. https://doi.org/10.1177/1087054715577135

Zisapel, N., \& Nir, T. (2003). Determination of the minimal clinically significant difference on a patient visual analog sleep quality scale. Journal of Sleep Research, 12(4), 291-298. https://doi.org/10.1046/j.0962-1105.2003.00365.x

Received: March 7, 2019

Accepted: June 16, 2019

Published: June 26, 2019 